\title{
Rates of Dimerisation of Substituted Benzoic Acids
}

\author{
JøRGEN RASSING, OLE ØSTERBERG and THOR A. BAK
}

Chemistry Laboratory III, H. C. Orsted Institute, University of Copenhagen, Copenhagen, Denmark

\begin{abstract}
The ultrasonic absorption coefficient of benzoic acid, $p$-chlorobenzoic acid, $p$-methoxybenzoic acid, $p$-fluorobenzoic acid, and $p$ methylbenzoic acid, dissolved in dimethylformamide, have been measured at $25^{\circ} \mathrm{C}$ using a pulse-technique. The concentrations of the acids ranged from 0.4 to $2.0 \mathrm{M}$ and the measurements were carried out at approximately 10, 30, 50, and $70 \mathrm{Mc}$. From these data the rate constants for the association and dissociation reactions were calculated, assuming that only a simple dimerisation occurs. The rate constants for dissociation of the dimers of the five acids range from $1.0 \times 10^{-8} \mathrm{sec}^{-1}$ to $2.7 \times 10^{-8} \mathrm{sec}^{-1}$ and those for association range from $1.9 \times 10^{-7} \mathrm{sec}^{-1} \mathrm{l} \mathrm{mole} \mathrm{m}^{-1}$ to $5.9 \times 10^{-7} \mathrm{sec}^{-1} \mathrm{l} \mathrm{mole}{ }^{-1}$. Hammets $\sigma \cdot \varrho$ relation is found to be valid with a value of $\varrho=0.64$ for the dissociation reaction and $\varrho=-0.91$ for the association reaction.
\end{abstract}

\begin{abstract}
A ssociated compounds dissolved in unassociated solvents show a characteristic excess ultrasonic absorption. From that absorption it is possible to calculate the rate constants for the association and dissociation reaction as shown by Maier and Rudolph.1,2 They studied the association of benzoic acid dissolved in tetrachloromethane and interpretated the relaxation time in terms of rate constants assuming 1) that the mole fraction of acid is very much smaller than unity 2) that only dimerisation occurs, and 3) that the majority of the acid exists as dimer. By measuring the ultrasonic absorption at $20 \mathrm{Mc}$ at different concentrations of acid and at different temperatures Maier and Rudolph obtained the rate constants for the dimerisation of benzoic acid. In a later paper Maier, Borucki, Dischler, Manogg and Rieseberg ${ }^{3}$ verified the results using the sound absorption of the solution at different frequencies.

To our knowledge the rate constants for the dimerisation of substituted benzoic acids are not available in the literature.

We have determined the rate constants for association and dissociation reactions of benzoic acid, $p$-chlorobenzoic acid, $p$-methoxybenzoic acid, $p$-fluorobenzoic acid, and $p$-methylbenzoic acid, dissolved in dimethylformamide, using the relaxation times at different concentrations of acid. Further-
\end{abstract}

Acta Chem. Scand. 21 (1967) No. 6 
more we have tested the Hammet equation for the association and dissociation reaction.

Dimethylformamide which we used as solvent has a strong tendency for forming hydrogen bonds and probably associates with the monomeric acid. Nevertheless the reaction may be represented by an equation of the following form, provided that only dimerisation occurs:<smiles>CC#CCC(=O)O[Hg]O[C@H](C)O</smiles>

where the asterisk indicates that there is a substituent in the benzene nucleus. The kinetics of the process can be written:

$$
\frac{\mathrm{d}\left[\mathrm{A}_{2}\right]}{\mathrm{d} t}=k_{21}[\mathrm{~A}]^{2}-k_{12}\left[\mathrm{~A}_{2}\right]
$$

where $k_{12}$ and $k_{21}$ are, respectively, the forward and the backward rate constants and $[x]$ indicates the molar concentration of $x$.

The absorption coefficient of the solution is described by the following equation,

$$
\frac{\alpha}{v^{2}}=\frac{A}{1+\left(v / v_{\mathrm{r}}\right)^{2}}+B+B^{\prime}
$$

where $\alpha$ is the absorption coefficient of the solution, $\nu$ is the frequency of sound, $v_{\mathrm{r}}$ is the relaxation frequency, $A$ is an arbitrary constant, and $\left(B+B^{\prime}\right)$ is the part of the absorption divided by the frequency squared due to the vibration and other processes occurring in the solution. This absorption differs from the absorption of the pure dimethylformamide by the term $B^{\prime}$, which at constant temperature is a function of the concentration of acid only. In this paper $B^{\prime}$ is approximated to zero which seems to be reasonable in view of the experimental results (see below).

The interpretation of the relaxation time given by Maier and Rudolph 1 can not be used in our case because the assumption that most of the acid exists as dimer is not valid. Neglecting that assumption we obtain the following equation:

$$
\left(2 \pi v_{\mathrm{r}}\right)^{2}=(1 / \tau)^{2}=8 k_{12} k_{21} c+k_{12}^{2}
$$

where $c$ is the total concentration of acid. Eqn. (4) gives the values of $k_{12}$ and $k_{21}$ from the relaxation times at different concentrations of the acid.

The effect of substituents on the rates of a side chain reaction of benzene derivatives can be described semiquantitatively by the Hammet equation ${ }^{4}$

$$
\log \frac{k}{k_{0}}=\sigma \varrho
$$


in which $k_{0}$ is the rate constant for the reaction of the unsubstituted compound and $k$ is the rate constant for the reaction of a compound which has a substituent meta or para relative to the reacting group. $\varrho$ is a quantity which depends only on the type of reaction. $\sigma$ depends only on the substituent and whether it is meta or para relative to the reacting group. Extensive tables of $\sigma$ and $\varrho$ values have been published ${ }^{5}$ but there are no $\varrho$-values for associationand dissociation reactions. Neither is it known if Hammets equation is valid at all for very fast reactions, although it seems very likely that it should be.

\section{EXPERIMENTAL}

The applied benzoic acids were the purest grade obtainable. The reaction was studied at $25^{\circ} \mathrm{C}$ in dimethylformamide because of the convenient solubility of the derivatives. The absorption coefficients of the solutions were measured at 9.90, 29.5, 49.5, and 68.6 Mc by means of a pulse-method described earlier. ${ }^{6}$ The concentration of acid was between 0.4 and $2.0 \mathrm{M}$. The absorption coefficient of pure dimethylformamide divided by the frequency squared, $B$ was found to be independent on the frequency in the frequency region we used. From the absorption data and eqn. (3) we calculated the best theoretical curves and relaxation frequencies using an iterative least squares method. (The standard deviations were smaller than 0.03 ). Fig. 1 a-e shows the results for different concentrations of each acid.
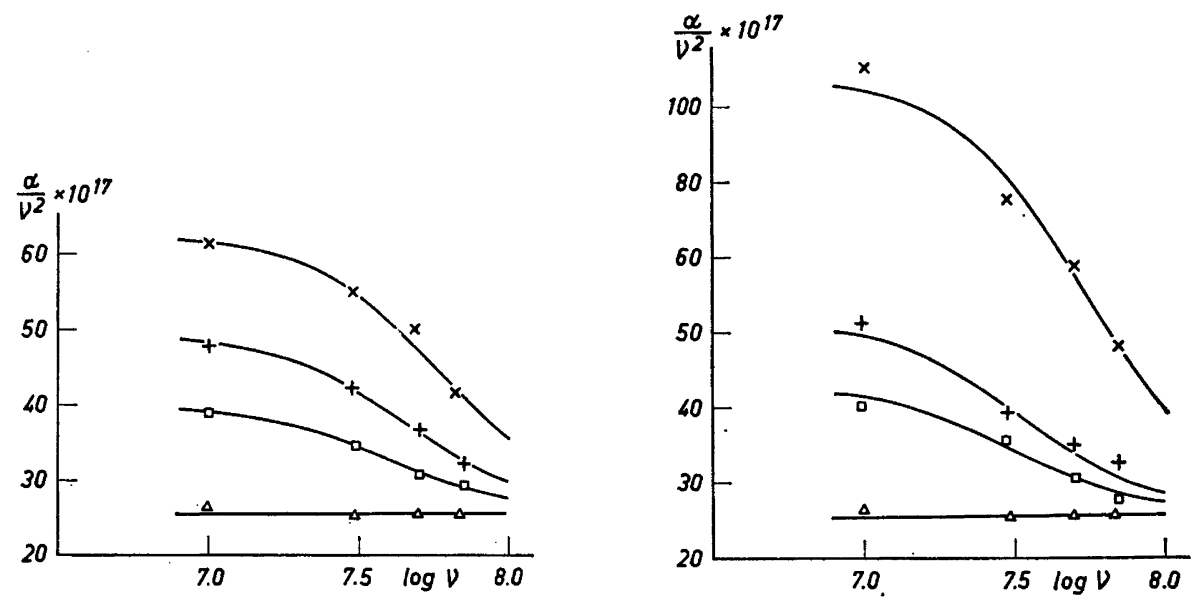

Fig. $1 a-e$ : The dependence of the absorption divided by the frequency squared on the logarithm of the frequency.

Fig. 1 a. Benzoic acid dissolved in dimethylformamide. $X: 1.93 \mathrm{M}$. + : $0.985 \mathrm{M}$.

$0.470 \mathrm{M} . \Delta$ : pure dimethylformamide.
Fig. 1 b. p-Methoxybenzoic acid dissolved in dimethylformamide. $x: 2.15 \mathrm{M}$. + : 1.22 M. $\square$ : 0.475 M. $\Delta$ : pure dimethylformamide. 


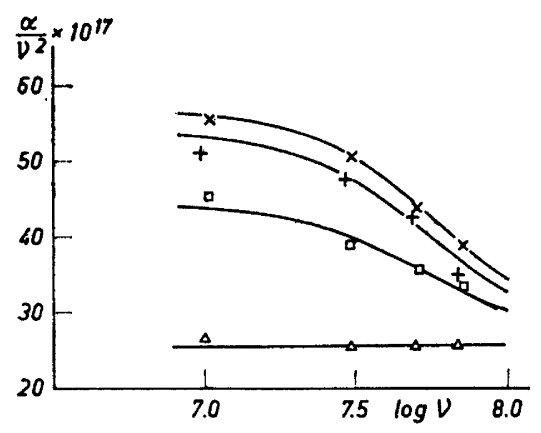

Fig. 1 c. $p$-Chlorobenzoic acid dissolved in dimethylformamide. $x: 1.43 \mathrm{M}$. $+: 1.10 \mathrm{M}$. $\square: 0.730$ M. $\triangle$ : pure dimethylformamide.

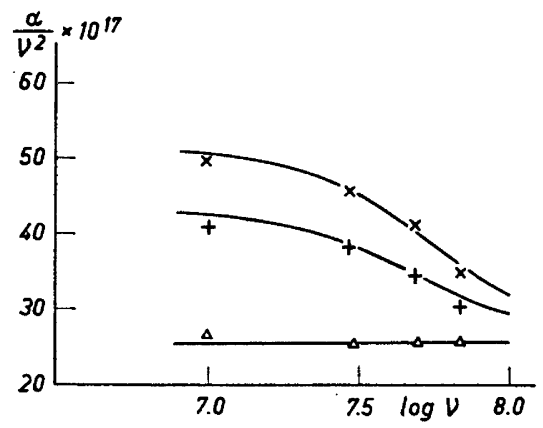

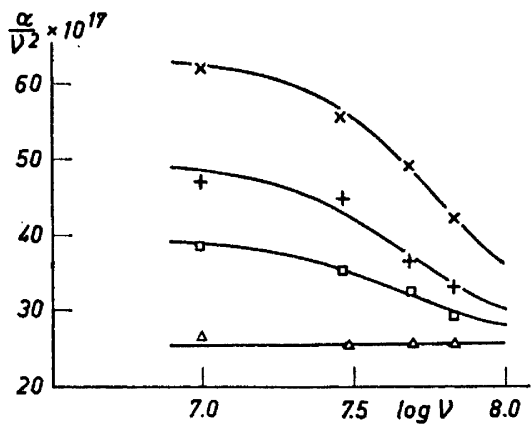

Fig. 1 d. p-Methylbenzoic acid dissolved in dimethylformamide. $\times: 1.63 \mathrm{M}$. $+: 0.642$ M. $\square$ : 0.362 M. $\triangle$ : pure dimethylformamide.
Fig. 1 e. p-Fluorobenzoic acid dissolved in dimethylformamide. $x: 1.02 \mathrm{M}$. $+: 0.500$

M. $\Delta$ : pure dimethylformamide.

\section{RESULTS}

It appears that the experimental values of $\alpha / \nu^{2}$ to a good approximation are linear functions of the logarithm of the frequency in the frequency region $30-70 \mathrm{Mc}$, in which we expect the relaxation frequencies to be located. From the slopes of these lines it is possible to estimate the values of $A$ without assuming $B^{\prime}$ to be zero (see eqn. (3)). The result is

$$
\left(\frac{\mathrm{d}\left(\alpha / \nu^{2}\right)}{\mathrm{d} \log _{10} v}\right)_{\nu=\nu_{\mathrm{r}}}=-\frac{A}{0.869}
$$

If we compare the values of $A$ obtained from eqn. (6) with the values of $A$ given by the best theoretical curves it is possible to test the approximation that $B^{\prime}$ equals zero. In Table 1 are listed the different values of $f$ defined as the ratio between the corresponding values of $A$. It is a check on the validity of the approximation that the variations of $f$ are not systematic as they would be if the value of $\left(B+B^{\prime}\right)$ is a function of the concentration of acid. Consequently the approximation that $B^{\prime}$ equals zero is valid within the accuracy with which we work. 


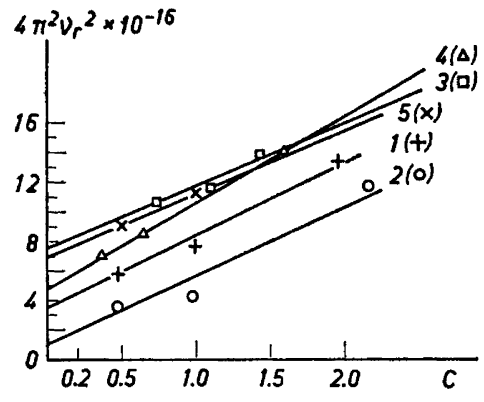

Fig. 2. The dependence of the relaxation frequency on the concentration of the different acids.

1: benzoic acid, 2: $p$-methoxybenzoic acid, 3: $p$-chlorobenzoic acid, 4: $p$-methylbenzoic acid, 5: $p$-fluorobenzoic acid.

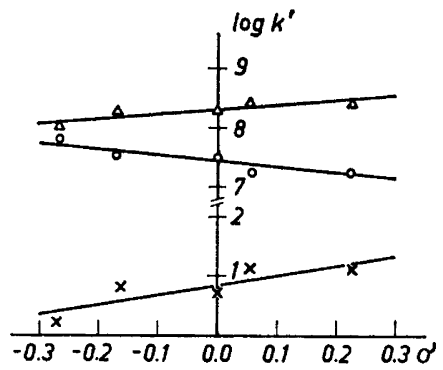

Fig. 3. The values of $\log k^{\prime}$ of the different acids plotted against the corresponding $\sigma$-values. $\log k^{\prime}$ is, respectively: $\triangle: \log k_{12}$, $0: \log k_{21}, \times: \log K$.

Fig. 2 shows the dependence of the relaxation frequency on the concentration of the different acids. Using eqn. (4) the rate constants listed in Table 2 are calculated. The equilibrium constants, $K$, are obtained from the ratio $k_{12} / k_{21}$.

Table 1. The values of $f$ (the ratio between corresponding values of A) calculated for each acid at three different concentrations.

\begin{tabular}{|l|l|l|l|}
\hline & \multicolumn{3}{|c|}{$f$} \\
\cline { 2 - 4 } & $c_{1}$ & $c_{2}$ & $c_{2}$ \\
\hline & 0.86 & 1.0 & 0.93 \\
$\mathrm{C}_{6} \mathrm{H}_{6} \mathrm{COOH}$ & 0.80 & 1.1 & 0.91 \\
$p-\mathrm{ClC}_{6} \mathrm{H}_{4} \mathrm{COOH}$ & 1.1 & 1.1 & 0.80 \\
$p \cdot \mathrm{CH}_{3} \mathrm{OC}_{6} \mathrm{H}_{4} \mathrm{COOH}$ & 1.0 & 0.91 & 1.2 \\
$p \cdot \mathrm{CH}_{3} \mathrm{C}_{6} \mathrm{H}_{4} \mathrm{COOH}$ & 1.0 & 1.0 & \\
$p-\mathrm{FC}_{6} \mathrm{H}_{4} \mathrm{COOH}$ & &
\end{tabular}

Table 2. The obtained values of the rate constants and the equilibrium constants.

\begin{tabular}{|c|c|c|c|}
\hline Compound & $k_{12} \times 10^{-8}$ & $\underset{\mathrm{sec}^{-1}}{k_{21}} \times 1 \mathrm{~mole}^{-1}$ & $\underset{1^{-1} \frac{K}{\text { mole }}}{K}$ \\
\hline $\begin{array}{l}\mathrm{C}_{6} \mathrm{H}_{5} \mathrm{COOH} \\
p-\mathrm{ClC}_{6} \mathrm{H}_{4} \mathrm{COOH} \\
p-\mathrm{CH}_{3} \mathrm{OC}_{6} \mathrm{H}_{4} \mathrm{COOH} \\
p \cdot \mathrm{CH}_{3} \mathrm{C}_{6} \mathrm{H}_{4} \mathrm{COOH} \\
p-\mathrm{FC}_{6} \mathrm{H}_{4} \mathrm{COOH}\end{array}$ & $\begin{array}{l}1.8 \\
2.7 \\
1.0 \\
2.2 \\
2.7\end{array}$ & $\begin{array}{l}3.4 \\
1.9 \\
5.9 \\
3.4 \\
2.0\end{array}$ & $\begin{array}{c}5.3 \\
14 \\
1.7 \\
6.5 \\
13\end{array}$ \\
\hline
\end{tabular}


Fig. 3 shows the values of $\log k^{\prime}$ for the different acids plotted against the corresponding $\sigma$-values, and it appears that the Hammet equation is valid within the accuracy of the experiments. The $\varrho$ values of the association and dissociation reactions are calculated from the slopes of the lines and are found to be, respectively, -0.91 and 0.64 .

A positive value of $\varrho$ indicates that the reaction is facilitated by low electron density at the reaction site and a negative value of $\varrho$ implies a reaction favored by high electron density. The $\varrho$-values quoted above are thus in agreement with intuitive notion that establishing a hydrogen bond to an oxygen atom is facilitated by a relatively high electron density around the oxygen atom.

\section{REFERENCES}

1. Maier, W. and Rudolph, H. D. Z. Elektrochem. 60 (1956) 944.

2. Maier, W. and Rudolph, H. D. Z. physik. Chem. (Frankfurt) 10 (1957) 83.

3. Maier, W., Borucki, L., Dischler, R., Manogg, P. and Rieseberg, H. Z. physik. Chem. (Frankfurt) 26 (1960) 27.

4. Hammet, L. P. J. Am. Chem. Soc. 59 (1937) 96.

5. Jaffè, H. H. Chem. Rev. 53 (1953) 191.

6. Kruus, P. and Bak, T. A. Acta Chem. Scand. 20 (1966) 231.

Received March 1, 1967. 